\title{
Examination of Alzheimer-Type Neurodegeneration in Mouse Trisomy 16 Neurons Maintained by Transplantation
}

\author{
J. Stoll ${ }^{1}$, A. Fine ${ }^{2}$, A. Balbo ${ }^{1}$, and S.I. Rapoport ${ }^{1}$ \\ ${ }^{1}$ Laboratory of Neuroscience, National Institute on Aging, Bethesda, MD 20892, USA \\ and ${ }^{2}$ Dalhousie University, Halifax, NS, Canada
}

Down's syndrome (DS, trisomy 21) results from an extra copy of human chromosome 21. Included in the phenotype of this syndrome is the development of neurofibrillary tangles and neuritic plaques that are characteristic of Alzheimer's disease (AD) (Wisniewski KE et al., Ann Neurol 1985; 17: 278-282). The hippocampus is an early and critical site of this pathology. Animal models would greatly aid the elucidation of processes underlying the neuropathology of $\mathrm{DS}$ and $\mathrm{AD}$. Murine trisomy 16 is a proposed genetic model for DS owing to the homology between mouse chromosome 16 and human chromosome 21 (Epstein CJ, Trends Genet 1985; 1: 129-134). To determine whether the trisomy 16 mouse could be a useful model for $\mathrm{AD}$, we investigated whether trisomy 16 brain tissue develops $\mathrm{AD}$ neurodegeneration and neuropathology. Because age is an important factor in the development of $\mathrm{AD}$ neurodegeneration and trisomy 16 mice do not survive past birth, neural tissue was maintained by transplantation so that the neurons might live long enough to express the $\mathrm{AD}$ phenotype. Trisomy 16 mice were produced by mating males doubly heterozygous for the Robertsonian translocations $\mathrm{Rb}(11.16) 2 \mathrm{H} / \mathrm{Rb}(16.17) 32 \mathrm{Lub}$ to C57B1/6 females. Hippocampal anlagen from embryonic day 15-17 fetal mice, trisomic for chromosome 16 or euploid litter-mates, was dissected free, cut into two or three pieces and taken up into a $10 \mu \mathrm{l}$ Hamilton syringe. A craniotomy was performed in host 6-8 week old female C57B1/6 mice and the tissue was injected stereotaxically into striatum or lateral ventricle. In some cases, hippocampal tissue was dissociated with trypsin prior to grafting (Fine A, Neurosci Lett 1991; 122: 4-8). Following up to fourteen months of survival in vivo, brains were fixed by perfusion with $4 \%$ paraformaldehyde, sectioned and the grafts were examined by standard histochemical techniques and by immunocytochemistry for senile plaques and neurofibrillary tangles of $\mathrm{AD}$ patients. Nissl-stained grafts appeared healthy with no areas of necrosis or degeneration. The grafts contained neuronal aggregates similar to the pyramidal- or the granule-cell layers of the normal adult hippocampus. No obvious morphological difference was detected between trisomic and control transplants. No evidence of neuropathology was found in thioflavin S- or silver-stained sections. The monoclonal antibody Alz-50 (provided by Dr. P. Davies, Albert Einstein College of Medicine, and Dr. H. Ghanbari, Abbott Lab.), which recognizes the paired helical filaments characteristic of $\mathrm{AD}$, did not stain trisomic or euploid grafts. However, frontal cortex from an $\mathrm{AD}$ patient, stained in parallel with the mouse sections, reacted strongly, thus indicating that the lack of staining in the mouse sections was not a failure of the immunohistochemical procedure. An antibody to $\beta$-amyloid peptide (R1280, provided by Dr. I. Lieberberg, Athena Neurosciences) revealed no $\beta$-amyloid accumulation in these grafts, whereas concurrent staining of $\mathrm{AD}$ frontal cortex demonstrated numerous plaques. We examined four trisomic en bloc grafts after one year of survival, and two grafts each after four, six and fourteen months using Alz-50 and R1280 antibodies; none showed AD-type immunoreactivity. Four dissociated cell trisomic grafts, after 18 months in vivo, did not stain with Alz-50. Further, antibodies against ubiquitin, 200 $\mathrm{kDa}$ subunit of neurofilament and tau, which stain neurofibrillary tangles, and antibodies 
against $\alpha 1$-antichymotrypsin, which stains plaques, demonstrated no $\mathrm{AD}$-type pathology in the trisomic or control grafts. In summary, we found no evidence of neurodegeneration or $\mathrm{AD}$ type immunoreactivity in grafts of trisomic hippocampal fragments in either the striatum or the lateral ventricle. The present results stand in contrast to the observation of neurofibrillary and amyloid immunoreactivity in solid grafts of trisomy 16 fetal hippocampus after only 4 months in vivo (Richards et al., EMBO J 1991; 10: $297-$ $303)$. While there are differences in grafting pro- tocol and mouse strain between these two studies that may lead to variations in expression of markers of cell pathology, it is clear that trisomy 16 tissue does not routinely develop any neuropathology comparable to that found in $\mathrm{AD}$. In conclusion, we report that mouse trisomy 16 neurons maintained up to 18 months by transplantation do not develop neuropathology or AD-type immunoreactivity as seen in DS, and thus do not provide a useful model for the development of $\mathrm{AD}$-type pathology. 

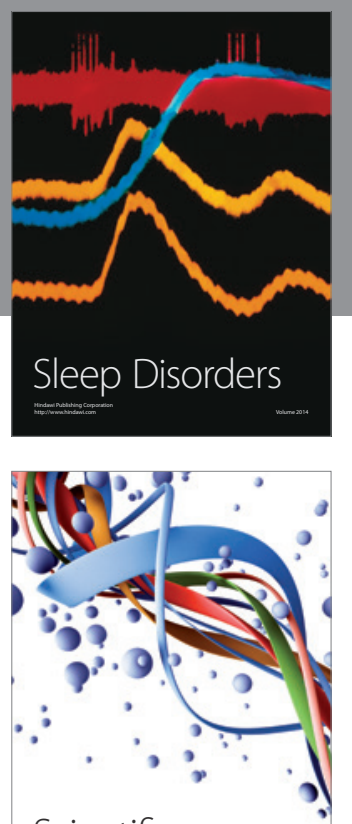

Scientifica
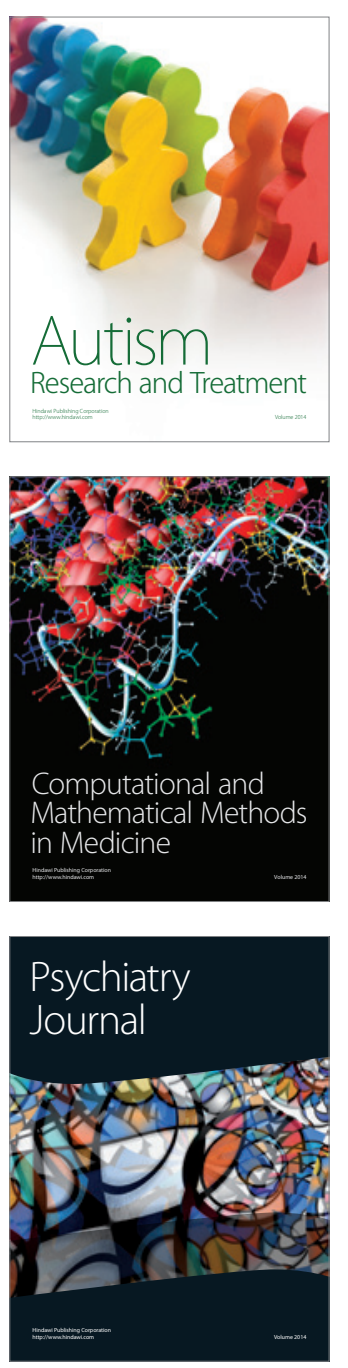
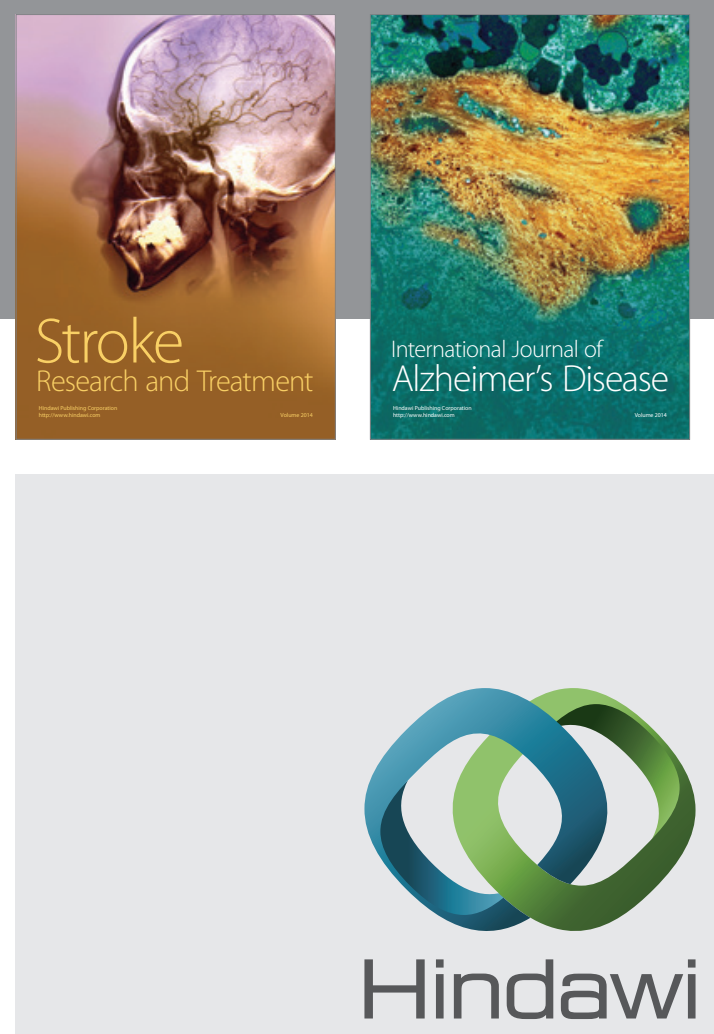

Submit your manuscripts at

http://www.hindawi.com
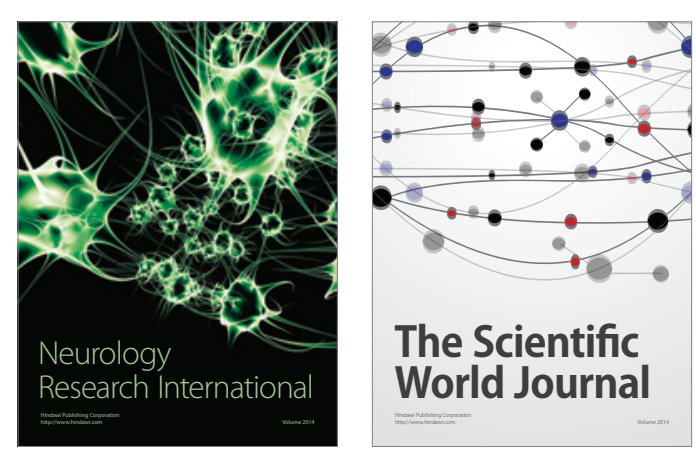

The Scientific World Journal

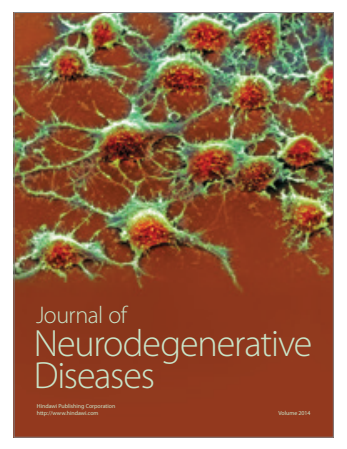

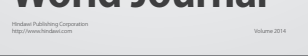

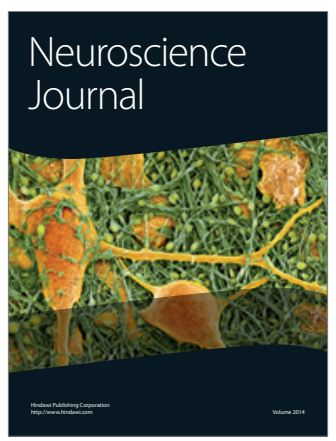

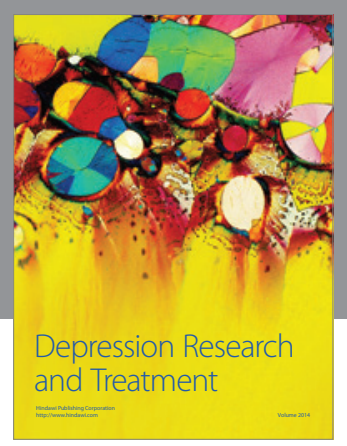
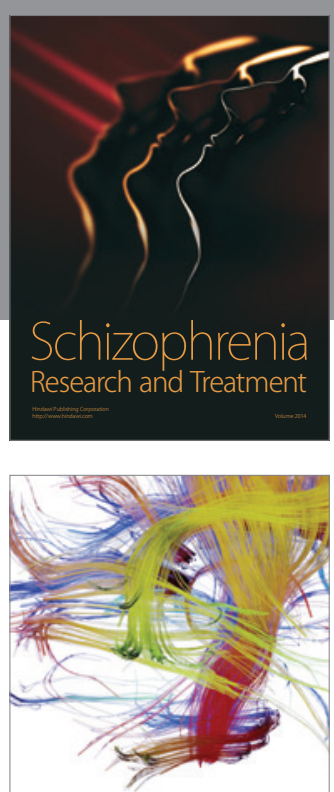

Brain Science

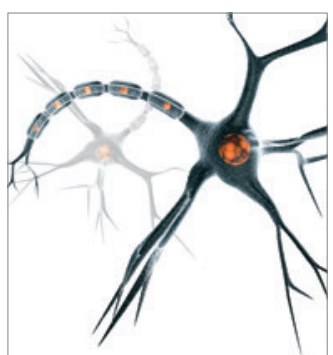

Neural Plasticity
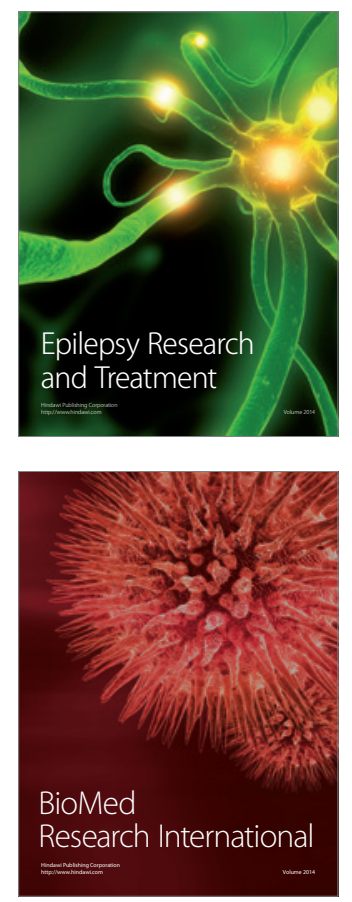

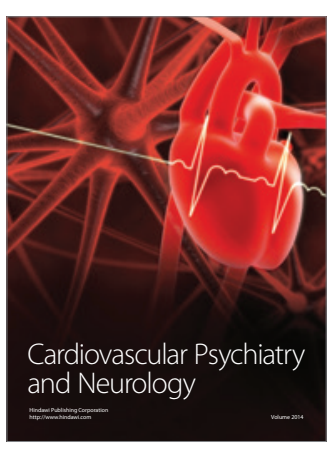

Parkinson's

Disease
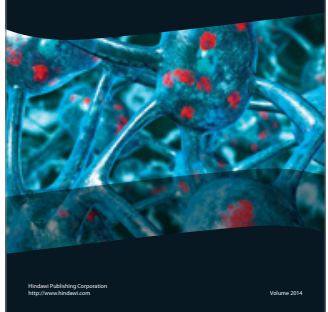